\title{
The effects of different arsenic species in relation to straighthead disease in rice
}

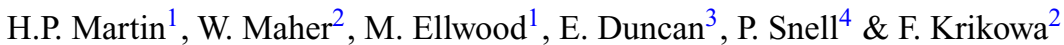 \\ ${ }^{1}$ Research School of Earth Sciences, Australian National University, Canberra, ACT, Australia \\ ${ }^{2}$ Institute for Applied Ecology, University of Canberra, Canberra, ACT, Australia \\ ${ }^{3}$ Future Industries Institute, University of South Australia, Adelaide, SA, Australia \\ ${ }^{4}$ Department of Primary Industries, Yanco Agricultural Institute, Orange, NSW, Australia
}

ABSTRACT: The effects of inorganic arsenic on plants, the mechanisms involved in the uptake and transport of arsenic and how inorganic arsenic enters food chains are well documented. Regulatory limits have been established to control the inorganic arsenic concentrations in certain foods including rice. There is, however, a knowledge gap with respect to dimethylarsenic concentrations. In this study rice was grown hydroponically and exposed to varying DMA concentrations. High levels of DMA were detrimental to rice plants whereby plants showed symptoms consistent with Straighthead disease, a disease that results in dramatic yield losses.

\section{INTRODUCTION}

Straighthead is a physiological disorder in rice that results in sterile florets and poorly developed panicles, leading to the head of the rice plant remaining upright when it reaches maturity (Dunn \& Dunn, 2012). Straighthead disease has the potential to generate in substantial yield declines. In Australia straighthead is expected to cost the rice industry in excess of \$1million per year. Although this cost could be much larger due to straighthead commonly being confused for cold-weather sterility. The exact cause of straighthead is unknown, however, studies have revealed a strong correlation between symptoms of the disease and elevated arsenic concentrations especially DMA within the plant and soil. The role arsenic plays with respect to straighthead disease is unknown, however, the presence of both inorganic (Rahman et al., 2008), and DMA (Yan et al., 2008) have been shown to increase the frequency and severity of straighthead symptoms in rice.

When investigating the relationship between arsenic speciation and total arsenic accumulation within rice plants, studies have found that the inorganic arsenic follows a hyperbolic pattern and levels out around $0.2 \mathrm{mg} \mathrm{kg}^{-1}$. DMA, however, does not follow this pattern and as a result has the potential to accumulate at much higher concentrations within the plant (Zhao et al., 2013). The uptake pathways and metabolism of DMA are largely unknown.

\section{METHODS/EXPERIMENTAL DESIGN}

\subsection{Seed germination and plant culture}

Rice seeds were surface sterilized utilizing a technique adapted by Kim (2005). Seeds were washed with

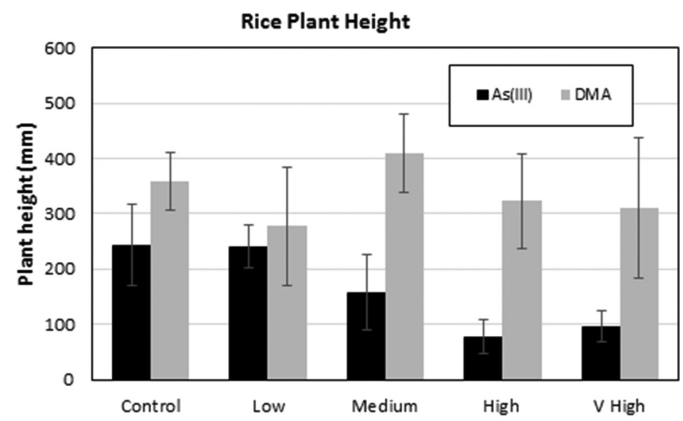

Figure 1. Effects of As(III) and DMA on plant height.

$10 \% \mathrm{H}_{2} \mathrm{O}_{2}$ for 10 minutes followed by $70 \%$ ethanol for 5 minutes followed by deionized water (DI). The seeds are than placed in DI water and transferred to an incubator at $30^{\circ} \mathrm{C}$ for $48 \mathrm{hr}$.

Seedlings were transplanted into purpose built hydroponic systems. The rice plants were grown using nutrient solution, exposing the rice to a half strength solution for the first 14 days, followed by full strength solution thereafter. The nutrient solution was aerated throughout the entire experiment, with the nutrient solution replaced weekly. The $\mathrm{pH}$ was kept between 5.5 and 5.8, adjusted daily using $1 \mathrm{~mol} \mathrm{~L}^{-1} \mathrm{HCL}$ and $1 \mathrm{~mol} \mathrm{~L}^{-1} \mathrm{NaOH}$.

Rice plants were exposed to either DMA or As(III) at four different concentrations; Low $0.8 \mu \mathrm{mol} \mathrm{L}^{-1}$, Medium $1.8 \mu \mathrm{mol} \mathrm{L}^{-1}$, High $3.5 \mu \mathrm{mol} \mathrm{L}^{-1}$ and Very High $6.7 \mu \mathrm{mol} \mathrm{L}^{-1}$. Each treatment was conducted in triplicate. Samples were grown for a total of 38 days.

Rice root and shoot samples where oven dried at $70^{\circ} \mathrm{C}$ until constant weight. Plants were digested using a low-volume microwave procedure developed. Approximately $0.2 \mathrm{~g}$ of dried plant samples into Teflon 
Growth of Rice Plants Exposed to $6.9 \mu \mathrm{mol} \mathrm{L}^{-1}$ of DMA

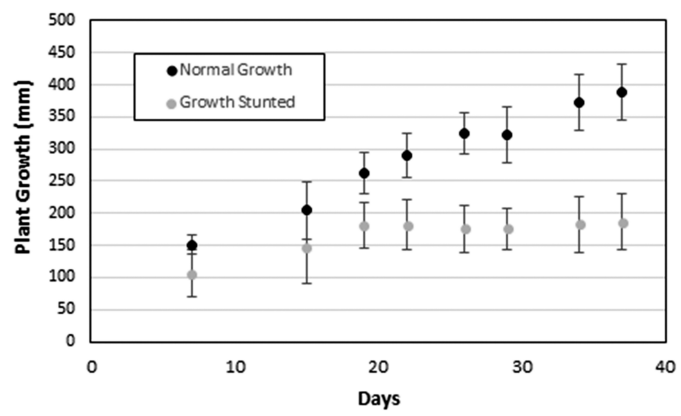

Figure 2. The grow rates of the plants of the of rice plants exposed to $6.7 \mu \mathrm{mol} \mathrm{L}^{-1}$, showing the different growth rates of plants within the same treatments.

closed digestion vessels with $2 \mathrm{ml}$ concentrated ultrapure nitric acid and $1 \mathrm{~mL}$ hydrogen peroxide. Samples were then microwaved using a preprogrammed digestion procedure. After digestion, samples are cooled at room temperature and the digests diluted to $10 \mathrm{~mL}$ with DI water for analysis by Inductively Coupled Plasms Mass Spectrometry (ICP-MS).

\section{RESULTS AND DISCUSSION}

\subsection{Plant growth}

Rice plants exposed to $\mathrm{As}(\mathrm{III})$ were observed to reduce in plant height with increasing As(III) exposure $(p<0.05)$. No significant differences in growth between the DMA treatments was observed, although there was significant variability in the data (Fig. 1).

\subsection{DMA effected rice plants}

The large variation in heights for DMA exposed plants was investigated further by looking at individual plant growth rates within treatments. For individuals exposed to $6.7 \mu \mathrm{mol} \mathrm{L}^{-1} \mathrm{DMA}$, these was a clear difference with two distinct populations; plants that exhibited normal growth and plants with stunted growth (Fig. 2). Plants were determined to have stunted growth if their growth rate was less than $50 \%$ of the control. Growth rate divergence occurred fourteen days after transplanting. Similar trends were observed across all the DMA treatments.

When the total arsenic concentrations between the two groups (Normal Growth and Stunted Growth) were compared, distinct differences were also observed (Fig. 3). The plants with stunted growth had more arsenic per gram of plant tissue with arsenic concentrations within the shoots nearly double of that of the plants with normal growth (Normal growth $15 \pm 8 \mu \mathrm{gg}^{-1}$, Stunted growth $43 \pm 8 \mu \mathrm{g} \mathrm{g}^{-1}$ ).

This indicates that DMA does not affect all plants, only a few in each treatment. Close inspection revealed that the reduced growth plants were not dead, even though growth had stopped. For most treatments, this
Total Arsenic Concentration in Rice Shoots

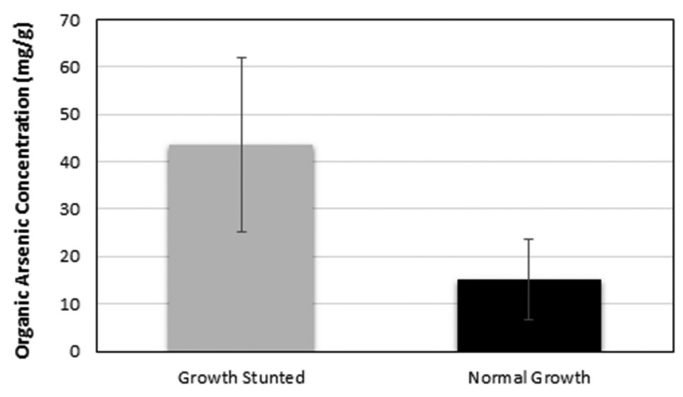

Figure 3. The concentration of arsenic in the rice shoots exposed to $6.7 \mu \mathrm{mol} \mathrm{L}^{-1}$ of DMA.

occurred between days 7 and 20 after transplanting. Rice plants exposed to DMA showed similar symptoms to plants affected by pathogens, with only a select few plants being affected.

\section{CONCLUSIONS}

This study highlighted that DMA has a larger effect on the rice plants than previously thought. While DMA is relatively non-toxic to humans, it still affects the plant differently than inorganic arsenic. There is, however, still considerable uncertainty regarding the uptake and metabolism of DMA. This study has highlighted that rice plants metabolize DMA differently to inorganic arsenic. With growing global concern of arsenic contamination in the environment, DMA could pose a real concern for crop health and productivity.

\section{ACKNOWLEDGEMENTS}

Ecochem UC for lab use and data analysis.

Australian Government Research Training Program ANU OCG Travel Scholarship.

\section{REFERENCES}

Dunn, B.W. \& Dunn, T.S. 2012. Influence of soil type on severity of straighthead in rice. Commun. Soil Sci. Plant Anal. 43(12): 1705-1719.

Kim, D.W., Rakwal, R., Agrawal, G.K., Jung, Y.H., Shibato, J., Jwa, N.-S., Iwahashi, Y., Iwahashi, H., Kim, D.H., Shim, I-S. \& Usui, K. 2005. A hydroponic rice seedling culture model system for investigating proteome of salt stress in rice leaf. Electrophorseis 26(23): 4521-4539.

Rahman, M.A., Hasegawa, H. \& Rahman, M.M. 2008. Straighthead disease of rice (Oryza sativa L.) induced by arsenic toxicity. Environ. Exper. Bot. 62(1): 54-59.

Yan, W.G., Agrama, H.A. \& Slaton, N.A. 2008. Soil and plant minerals associated with rice straighthead disorder induced by arsenic. Agron. J. 100(6): 1655-1661.

Zhao, F.J., Zhu, Y.G. \& Meharg, A.A. 2013. Methylated arsenic species in rice: geographical variation, origin, and uptake mechanisms. Environ. Sci. Technol. 47(9): 3957-3966. 